\title{
Die Ko-Konstitution von Struktur und Akteur: ein altes Problem aus Sicht kritischer Normenforschung
}

\author{
Eva Herschinger und Frank Sauer
}

\section{Einleitung}

Das Gros der heutigen Normenforschungsliteratur in den Internationalen Beziehungen (IB) ist bestimmt durch den (meta-)theoretischen Rahmen, den der Sozialkonstruktivismus Wendtscher Prägung abgesteckt hat (vgl. vor allem Wendt 1999). Dessen Verdienst ist es, von rationalistisch-materialistischen Paradigmen abweichende ontologische Prämissen in den IB häufig zusammengefasst als die Bedeutung von Ideen, Werten und Normen für die Konstruktion sozialer Realität - salonfähig gemacht zu haben. Mit dieser Entwicklung ging einher, dass sozialkonstruktivistische Normenforschung stets betonte, AkteurInnen und Strukturen stünden in einem ko-konstitutiven Verhältnis zueinander. In Abgrenzung zum bis dato bestehenden IB-Theoriekanon war mit dem Sozialkonstruktivismus insofern die Hoffnung auf eine konkrete Innovation verbunden: Das Augenmerk sollte endlich dezidierter und systematischer auf Strukturgenese und Strukturwandel durch Akteurshandeln, also das fortlaufende Wechselspiel zwischen (Fähigkeit zu und Notwendigkeit von) Handlungen in der sozialen Realität durch AkteurInnen und den diese Handlungsentscheidungen umgebenden strukturellen Zwängen, gerichtet werden können. ${ }^{1}$

Allerdings, so zeigte sich in der weiteren Entwicklung der sozialkonstruktivistischen Normenforschungsliteratur, kam die Bedeutung von Agency (Kärger et al 2017) - unter der wir im Folgenden die oben beschriebene Fähigkeit zu und Notwendigkeit von Handlungen in der sozialen Realität durch AkteurInnen verstehen - und damit der Gedanke der KoKonstitution nie voll zur Entfaltung (Bucher 2014). Für diese Entwicklung sind aus unserer Sicht zwei Gründe zentral, der erste theoretisch, der zwei-

1 Für hilfreiche Kommentare zu früheren Versionen des Beitrags bedanken wir uns bei den HerausgeberInnen, den TeilnehmerInnen des 4. Workshops des Netzwerks Kritische Normenforschung, den TeilnehmerInnen der IB-Kolloquien an der Universität der Bundeswehr München und am Geschwister-Scholl-Institut der LMU München sowie bei Alexander Spencer und Stephan Stetter. 
te methodologisch und forschungspraktisch. Ersterer ist die handlungstheoretische Inkonsistenz der Logik der Angemessenheit, die dem Großteil der sozialkonstruktivistischen Normenforschung zu Grunde liegt. Der zweite Grund ist darin zu suchen, dass der Wendtsche Sozialkonstruktivismus zwar das Feld für neue ontologische Prämissen aufschloss, zugleich aber weite Teile der in den IB fest verankerten positivistischen Epistemologie übernahm (siehe den Beitrag von Loges in diesem Band).

Um der Bedeutung von Agency im Verhältnis AkteurIn-Struktur besser Rechnung zu tragen, stellen wir in diesem Beitrag einige alternative Überlegungen zur Idee der Ko-Konstitution an, die wir einleitend kurz skizzieren, im weiteren Vorlauf dann ausführlicher entfalten und schließlich empirisch illustrieren möchten.

Am Anfang unserer Überlegungen stehen zwei geläufige Annahmen. Erstens umfasst der Begriff Diskurs in unserem Verständnis alle Praktiken, Bedeutungen und vor allem auch soziale Normen, die eine Gemeinschaft spezifischer AkteurInnen formen und prägen (Howarth 2000: 5). Insofern sind Diskurse soziale (Bedeutungs-)Strukturen, die beständig genutzt werden (müssen): Ein Diskurs ist eine "structure of meaning-in-use (Weldes/ Saco 1996: 373). ${ }^{2}$ Zweitens, ohne AkteurInnen, die sich in ihrem (Sprech-)Handeln auf diese "structure of meaning-in-use sen), sind soziale Strukturen bedeutungslos. AkteurInnen können also ohne durch Diskurs vermittelte, sinnstiftende soziale (Norm-)Strukturen (auf die sich ihr Handeln affirmativ oder negativ bezieht) nicht sozial sinnvoll handeln, und besagte Strukturen sind ohne handelnde AkteurInnen umgekehrt bedeutungslos bzw. in sozialem Sinne buchstäblich nicht existent. Kurz, Relation (Hofferberth/Weber 2015: 85) bildet den Ausgangspunkt bei sämtlichen Betrachtungen der Ko-Konstitution von AkteurInnen und Strukturen sowie der Funktion von Agency als Transmissionsriemen.

Ausgehend von diesen vermutlich unstrittigen Grundannahmen schlagen wir als nächsten Schritt eine Kombination aus Elementen pragmatistisch inspirierter Handlungstheorie und poststrukturalistisch informierter Diskurstheorie vor, um das Wechselspiel von AkteurIn und Struktur genauer in den Blick zu nehmen. Als Dreh- und Angelpunkte dienen uns die den beiden Theorien entlehnten - und als ebenfalls im beständigen, sich gegenseitig ermöglichenden Wechselspiel zu verstehenden - Konzepte Dis-

2 Um es noch deutlicher zu formulieren: Diskurse und Strukturen verwenden wir hier synonym, verzichten jedoch zugunsten der Lesbarkeit auf ein beständiges Nennen beider Begriffe. Vgl. für dieses Vorgehen beispielhaft Nonhoff/Gronau (2012: 121). 
lokation (als das stets lauernde Moment des Bruchs aufgrund einer nie abgeschlossenen Diskursstruktur) und Krise (als Abweichung von Routinen in Akteurspraktiken und als kreatives Moment). Sie verweisen darauf, dass weder Struktur noch Agency jemals als abgeschlossen zu betrachten sind; vielmehr konstituieren die Verbindungen, die zwischen ihnen entstehen, beide (in ihrer jeweiligen, temporär stabilen, stets prozesshaften Gestalt). Ko-Konstitution wird von uns also, stark verknappt und bezogen auf die hier im Fokus stehende Normforschung formuliert, als die Relation zwischen Normstruktur und Agency verstanden, durch die die Bedeutung von ersterer und letzterer bestimmt wird. Diese noch recht abstrakten Überlegungen entschlüsseln wir im weiteren Verlauf dieses Beitrags.

Zunächst gilt es jedoch darauf hinzuweisen, dass die Betonung des prozesshaften Wechselspiels von Dislokation und Krise mit Blick auf unsere weiteren Ausführungen natürlich ein Henne-Ei-Problem aufwirft. Analytisch, und zu Präsentationszwecken, müssen wir in diesem Papier das trennen, was wir eigentlich stets zusammendenken möchten. So ist also explizit keine Reihung zwischen den beiden vorgenommen, wenn wir unser noch ausführlich zu entwickelndes Kernargument zum ko-konstitutiven Prozess zwischen Akteur und Struktur und der Bedeutung von Agency in dieser Form knapp zusammenfassen: Diskurse sind dislozierbar durch soziale Prozesse, die im bestehenden Diskurs nicht gedeutet bzw. von AkteurInnen gemeistert werden können. Aus Akteursperspektive kann dies eine Krise bedeuten, also das Infragestellen von auf bisherigen Normstrukturen basierenden Praxisroutinen, wodurch neue Spielräume eröffnet (erzwungen) werden, in die kreatives Handeln vorstößt - was wiederum (auf mittlere und längere Sicht) neue Normstrukturen evozieren und bereits bestehende dislozieren kann (und so weiter). Sowohl Dislokationen und Krisen sind somit nicht zwangsläufig als singuläre Momente mit Ereignis-Charakter zu verstehen, sondern können sich schleichend entwickeln - ein Grund mehr, weswegen ein geschärfter Blick auf die Relationen von Akteur und Struktur nötig ist, um derartige Prozesse präzise nachzuzeichnen.

Die von uns vorgeschlagene Perspektive erlaubt es, sowohl einem statischen Strukturbegriff als auch der Vernachlässigung von Agency entgegenzuwirken und so normativen Wandel über beide Ebenen - Akteur und Struktur - besser erfassen zu können. Unsere Perspektive erleichtert zudem das Formulieren neuer Forschungsfragen: danach, wie genau Normen entstehen, was sie beinhalten, wie sie hinterfragt werden, wie sie wieder verschwinden - und nicht zuletzt, wie und durch was sie im Rahmen von Akteurshandeln ersetzt werden. Dementsprechend erschließt der hier vorgeschlagene Fokus auf die Momente der Krise und der Dislokation kritisches Potenzial, da sowohl Widerstand gegen als auch Reifizierung von be- 
stehenden Normstrukturen offengelegt und systematisch hinterfragt werden können. Letztlich wollen wir hier vor allem ein Vokabular entwickeln, das dem ko-konstitutiven Verhältnis von AkteurIn und Struktur besser Rechnung trägt und der bisherigen Normenforschung eine alternative Forschungspraxis zur Seite zu stellen erlaubt und für diese, so hoffen wir, Anknüpfungspunkte bieten kann.

Beginnend mit dem nächsten Abschnitt nehmen wir dazu zunächst eine detailliertere Problematisierung des derzeitigen Rahmens sozialkonstruktivistischer Normenforschung in zwei Schritten vor. Wir kritisieren erstens eine theoretische Blindstelle und diskutieren zweitens die methodologischen und forschungspraktischen Schwachpunkte gängiger Normenforschung, um die Vernachlässigung von Agency aufzuzeigen. Erneut in zwei Schritten vorgehend, legen wir daraufhin zuerst unsere Konzeption von Krise und Dislokation als den Kernelementen eines handlungs- und diskurstheoretisch informierten Alternativzugriffs auf die Ko-Konstitutivität des AkteurIn-Struktur-Verhältnisses ausführlich dar und diskutieren dann die sich daraus ergebenden methodologischen Implikationen. Anschließend illustrieren wir das kritische Potential einer dementsprechend unterfütterten Normenforschung kurz an der Entwicklung des internationalen Drogenprohibitionsregimes, gefolgt von einer Schlussbetrachtung.

\section{Problematisierung sozialkonstruktivistischer Normenforschung}

\subsection{Erster Schritt: Theorie}

Gemäß der Standarddefinition in der sozialkonstruktivistischen Normenforschung sind Normen "collective expectations for the proper behavior of actors with a given identity (Katzenstein 1996: 5). Normen bestimmen das Verhalten von AkteurInnen, indem sie eine Erwartungshaltung formulieren, wie sich eine AkteurIn in einer konkreten Situation verhalten soll. Soll, denn Normen sind "prescriptions for action in situations of choice, carrying a sense of obligation, a sense that they ought to be followed « (Chayes/Chayes 1994: 65, Hervorh. dort). In der konstruktivistischen Normenforschung ist also zentral, dass Normen für eine spezifische Gruppe von AkteurInnen relevant sind, d.h. für eine Gruppe, die über eine gemeinsame Identität verfügt - die wiederum von den entsprechenden Normen geprägt ist. Insofern erklären uns Normen, wie bestimmte AkteurInnen sich konstituieren, sie drücken aus, was bzw. wer diese AkteurInnen sind, zu welcher Gemeinschaft sie gehören und welche Rolle diese AkteurInnen spielen. Vor allem erklären Normen gemäß des Mainstreams der 
sozialkonstruktivistischen Normenforschung aber, warum AkteurInnen so handeln, wie sie handeln. Sie agieren demzufolge entlang der für sie gültigen Logik der Angemessenheit (grundlegend: March/Olsen 1998: 951-952).

Jedoch lässt diese an sich schlüssige Argumentation zu wünschen übrig, wenn es um die Entstehung neuer Normen geht. Zwar können gemäß gängiger Normenforschung AkteurInnen - norm entrepreneurs (grundlegend: Finnemore/Sikkink 1998) - Normen hervorbringen und ihre Verbreitung stärken (beispielsweise durch Institutionalisierung und Internalisierung) oder schwächen (beispielsweise durch Hinterfragen oder Widerstand), ${ }^{3}$ ebenso entstehen Normen durch Interaktion zwischen AkteurInnen (Hurrell 2002: 142-143). Allerdings liegt an dieser Stelle die wesentliche theoretische Inkonsistenz der Angemessenheitslogik, aus der eine strukturelle Schlagseite resultiert.

So kritisiert Ole Jacob Sending Martha Finnemore und Kathryn Sikkinks Argumentationsgang, nach dem »for new norms to emerge and become accepted by others, norm entrepreneurs or advocates have to act explicitly inappropriately« (2002: 460, Hervorh. dort). Da aber in Sozialstrukturen eingeschriebene Normen konstitutiv für Akteursidentitäten sind und diese angemessen handeln lassen, bleibt offen, wie und wann ein solch singulärer Akt der schöpferischen inappropriateness überhaupt zustande kommen soll. Wie Normen entstehen wird also recht bequem auf die Denkfigur des norm entrepreneurs ausgelagert. Theoretisiert wird es jedoch nicht weiter, die transformative capacity of agency bleibt somit unterbelichtet. Dementsprechend wies Benjamin Herborth in seiner Kritik am via media Konstruktivismus à la Wendt darauf hin, dass Strukturen im Sozialkonstruktivismus zwar als konstitutiv für Identitäten und die Bedingungen der Möglichkeit für Agency absteckend verstanden werden, umgekehrt der konstitutive Effekt der Agency aber vernachlässig bleibt:

"Die zentrale Innovationsleistung der ’konstruktivistischen Wender, die klassische Kausallogik um eine konstitutionstheoretische Perspektive zu erweitern, wird somit halbiert: [Worin] die konstitutiven Effekte sozialen Handelns bestehen, bleibt ausgeblendet. Die so halbierte Konstitutionslogik ist, gerade wegen dieser Asymmetrie, dann nicht mehr in der Lage, sozialen Wandel auf den Begriff zu bringen« (Herborth 2004: 62).

3 In diesem Zusammenhang wird auch von norm challengers gesprochen, vgl. Heller et al. (2012). 
Mit Blick auf Alexander Wendts Artikel Why a World State is Inevitable spricht Vaughn Shannon aufgrund eben dieser stiefmütterlichen Behandlung von Agency sogar von einer "violation of the constructivist project" (Shannon 2005).

Wir behaupten hier nicht, dass die Umstrittenheit (und somit das Entstehen und Verschwinden) von Normen in der sozialkonstruktivistischen Forschung nicht prinzipiell bereits anerkannt wurde. ${ }^{4}$ Jedoch liegt hier, so unser Argument, noch viel und bisher ungehobenes kritisches Potenzial das zu seiner Entfaltung aber einer expliziteren und systematischeren Einbeziehung von Agency bedarf. Denn die Frage, wie es möglich ist, dass Normen umstritten sein können und welcher Angemessenheitslogik unangemessen handelnde norm entrepreneurs denn folgen, wenn es nicht (mehr) die kollektiv erwartete und somit die normal gültige ist, all dies fällt eben in die beschriebene theoretische Blindstelle. Und auch weil Normen sich nicht ins Nichts verflüchtigen, sondern, gleich ob bestritten, verletzt oder ignoriert, von AkteurInnen stets ersetzt werden, müsste Agency systematisch in den Blick genommen werden, wenn man die Frage nach dem $E r$ setzt wie und wodurch? genauer beantworten wollte.

In der Vergangenheit wurde als Reaktion auf diese und ähnliche Fragen vorgeschlagen, Normen so zu konzeptualisieren, dass ihre Definition unabhängig von den ihnen zugeschrieben Effekten ist. Andere suchten den Zugriff auf normativen Wandel in der Analyse des Zusammenspiels von internationalen und regionalen, nationalen (weniger lokalen) Normen (vgl. unter anderen Kier 1997; Legro 1997); in jüngster Zeit wurde entgegen diesen theoretischen Konzeptualisierungen argumentiert, dass dies vor allem eine empirische Frage sei (Heller et al. 2012: 283-284). Ein hand-

4 Vgl. Badescu/Weiss (2010); Heller et al. (2012); Liese (2009); Picarelli (2009). Zur Erosion von Normen siehe vor allem McKeown (2009); Rosert/Schirmbeck (2007), siehe auch Panke/Petersohn (2012); wobei die dem letztgenannten Beitrag zu Grunde liegende Forschungslogik die hier thematisierten Probleme mit verursacht hat, wie wir im nächsten Abschnitt thematisieren werden.

Nicole Deitelhoff schlägt vor, das Entstehen von Normen bzw. deren Entwicklung nach Habermas idealtypisch als durch freien Austausch von Argumenten in einem rationalen Diskurs erwirkte Überzeugungsprozesse zu konzeptualisieren - sie versteht dies aber eher als Absatzbewegung zu rational-choice-Ansätzen des »institutional design « und fokussiert primär auf die Ebene des Diskurses, auf »changes in positions, normative frames, and the institutional setting over time in search of correlations that correspond to the hypothesis derived from the discourse approach while simultaneously controlling for alternative factors" (Deitelhoff 2009: 61). Die hier eingeforderte, handlungstheoretische Unterfütterung der Normenforschung hinsichtlich der konstitutiven Funktion von Agency steht somit weiterhin aus. 
lungstheoretisch unterfütterter Fokus auf Agency wurde bisher nicht gelegt.

So bleiben AkteurInnen, zugespitzt formuliert, nach dem aktuellen Stand der Forschung theoretisch weitgehend doch nur Befolgungsautomaten der Angemessenheitslogik (Sending 2002: 455, 458). Das prädestiniert, wie oben bereits angedeutet, zu Forschung mit einem Interesse daran, wie bestehende Normstrukturen Akteurshandeln bewirken, also dieses zu erklären. Und darin liegt unserer Ansicht nach der zweite Teil des Problems.

\subsection{Zweiter Schritt: Methodologie und forschungspraktische Implikationen}

Die Kombination der handlungstheoretischen Blindstelle sozialkonstruktivistischer Normenforschung, die einen theoretischen Fokus auf Strukturen befördert, mit der Bewahrung einer positivistischen Methodologie, so unser Argument weiter, erhöhte die Schwierigkeiten eines Augenmerks auf Agency. Die Schwierigkeit eines Zugriffs auf Normen via positivistische Methoden und einer auf Kausalzusammenhänge abzielenden Methodologie haben Friedrich Kratochwil und John Gerad Ruggie längst beschrieben:

»Norms may sguider behaviour, they may rinspire behaviour, they may >rationalize< or 'justify< behaviour, they may express >mutual expectations about behaviour or they may be ignored. But they do not effect cause in the sense that a bullet through the heart causes death or an uncontrolled surge in the money supply causes price inflation « (Kratochwil/Ruggie 1986: 767).5

Hier wird deutlich, weswegen das Vernachlässigen von Agency als der Preis beschrieben werden kann, den die konstruktivistische Normenforschung Wendtscher Prägung zahlte, als sie eine via media mit Blick auf den epistemologischen Status von Normen beschritt. Deren Anschlussfähigkeit liegt darin, dass das Verhältnis von AkteurIn und Struktur eben zur gleichen Zeit unabhängig und abhängig, kausal und konstitutiv sein könne. Zu nennen ist hier etwa Emmanuel Adlers Überlegung einer "social causality« (Adler 1997: 329), die eine Alternative sei, um einen spezifisch konstruktivistischen Kausalzusammenhang zwischen Akteurshandeln und Struktur-

5 Davis (2005: 134-135) weist folgerichtig darauf hin, dass gerade weil Normen Verhalten nicht in dieser Form kausal bewirken, sie kontrafaktische Gültigkeit beanspruchen können. 
wirkung herzustellen. ${ }^{6}$ Auch fordern Wendt und andere critical realists wie Milja Kurki (2006) in diesem Sinne die Abkehr von der Hume'schen Logik linearer Kausalität in den IB.

Doch trotz - oder gerade wegen - solch umstrittener Teilfragen ist klar erkennbar, dass die Mainstream-Normenforschung in der kausalen Binnenlogik verhaftet und das positivistische Paradigma intakt geblieben ist. So ist sie zwar in der Lage, die Wechselwirkung zwischen AkteurInnen und Normen nachzuzeichnen (vgl. Finnemore/Sikkink 1998; Klotz 1995; Risse et al. 1999), doch liegt der forschungspraktische Fokus stets auf der Wirkung der Norm auf den Akteur oder die AkteurIn. Viele AutorInnen des konstruktivistischen Mainstreams stellen hier folglich gewohnheitsmäßig auf einen kausalanalytischen Zusammenhang ab. ${ }^{7}$

Wenn also Nina Tannenwald (1999; 2005a; 2005b; 2007) $)^{8}$ erklärt, warum die USA nach 1945 wiederholt vom Nuklearwaffeneinsatz in Kriegen abgesehen haben, dann hat das nach konstitutionslogischem Verständnis zwar auch mit der identitätsstiftenden Funktion des »nuklearen Tabus« (Tannenwald 1999) zu tun, also dem Selbstverständnis der USA als "zivilisierter Nation«, wird aber vorrangig eben doch auf die kausale Wirkung des nuklearen Tabus zurückgeführt, dessen regulierender Effekt auf AkteurInnen die Erzeugung eines normangemessenen Verhaltens - hier der Nichtgebrauch von Nuklearwaffen - ist. ${ }^{9}$ In diesem Sinne praktiziert der sozialkonstruktivistische Mainstream der Normenforschung in den IB einfach das übliche positivistische Denken weiter (Hofferberth/Weber 2015: 81).

Diese Forschungspraxis hat viel Kritik auf sich gezogen (wie bereits erwähnt auch von Sozialkonstruktivisten), denn ganz im Sinne von Kratochwil und Ruggie wurde die Möglichkeit von kausallogischer Normenforschung von einigen Zwischenrufern immer wieder grundsätzlich mit der Begründung verneint, dass sich Normen als soziale Konstrukte einer positivistischen Forschungslogik entziehen. Und um einem Missverständnis

6 Andere Vorschläge finden sich beispielsweise bei Klotz (1995) und Yee (1996).

7 Vgl. neben den oben bereits genannten Finnemore/Sikkink (1998); Katzenstein (1996); Klotz (1995); Risse et al. (1999); Tannenwald (2007).

8 Nina Tannenwald wird hier unter anderem auch deswegen als Beispiel herangezogen, weil sie mit Artikeln zum nuklearen Tabu in International Security (2005b), International Organization (1999) und einem Buch bei Cambridge University Press (2007) als stellvertretend für das Ankommen der Normenforschung im Mainstream der IB-Spitzenforschung gelten kann. Im Artikel Ideas and Explanation: Advancing the Theoretical Agenda (2005a) legt sie ihr Vorgehen programmatisch dar.

9 Die struktur- und kausallogische Schlagseite dieser Perspektive wird vollends offenkundig, wenn bei Tannenwald explizit auf Designing Social Inquiry von King, Keohane und Verba (1994) als Referenzrahmen verwiesen wird. 
vorzubeugen: Wir werfen Forschungen wie der hier als Beispiel um das nukleare Tabu bemühten selbstverständlich nicht ihr Erkenntnisinteresse vor - zumal sie weitreichende und wichtige Ergebnisse und Einsichten erbracht und die Disziplin immens bereichert haben. Dennoch lässt sich aus unserer Sicht der folgende Befund aufrechterhalten: Ausgerüstet mit dem positivistischen Denkzeugkasten überrascht es nicht sonderlich, wenn der Blick von NormenforscherInnen (gerade mit einem theoretisch ohnehin von strukturellem Übergewicht gekennzeichneten AkteurIn-Struktur-Verhältnis im Kopf) über kurz oder lang an den verhaltensregulierenden Strukturen hängenbleibt, ergo die oben angeführte Grundsatzkritik an auf Kausalität abzielender Normenforschung schlichtweg forschungspraktisch nie verfing. Daher, so unser Argument, wurde die ursprünglich explizit betonte Ko-Konstitutivität von AkteurIn und Struktur in der bestehenden Forschungspraxis bis heute nicht systematisch eingelöst (Hofferberth/ Weber 2015: 79). Dem wollen wir nun durch das Anlegen einer alternativen Perspektive begegnen.

\section{Formulierung eine handlungs- und diskurstheoretisch informierten Alternativperspektive}

\subsection{Erster Schritt: Dislokation und Krise}

Aus unserer Sicht passen ein von pragmatistischer Handlungstheorie inspiriertes Konzept von Agency und ein von poststrukturalistisch informierter Diskurstheorie inspiriertes Verständnis von Struktur zusammen, da beide durch ihre Betonung der Instabilität von Bedeutungen und Praktiken der Kontingenz sozialer Realität gerecht werden. Mehr noch: das Einbeziehen von handlungstheoretischen Überlegungen erlaubt es, die Diskurstheorie zu präzisieren mit Blick darauf wie Prozesse des Handelns, des Entscheidens konkret ablaufen. Am Ende unserer Überlegungen steht so ein theoretisch gehaltvolleres Konzept des Verhältnisses von AkteurIn und Struktur, als es Diskurstheorie oder pragmatistische Handlungstheorie allein bieten können. So wird der Überbetonung einer strukturähnlichen Wirkung von Normen entgegenwirkt und die Rolle von Agency systematisch in den Blick genommen.

Diskurstheoretisch informierte LeserInnen mögen sich spätestens jetzt zwei Fragen stellen. Erstens: Wie viel vom Subjektbegriff steckt in unse- 
rem oder unserer AkteurIn? ${ }^{10}$ Dazu gilt es zunächst festzuhalten, dass der »Akteurshut« (Nonhoff/Gronau 2012: 128), den wir uns hier aufsetzen, ein in der Subjekt-Wolle gefärbter Hut ist. Denn was wir hier aus dem Begriff des Subjekts in den Akteur oder die Akteurin hineintragen wollen, ist das Moment des Unterworfen-Seins, welches das Subjekt auszeichnet, neben dem Gestaltenden, dem Sich-Ermächtigen. So ist in unserem Verständnis Agency als kreatives (gestaltendes) Handeln trotz Unterwerfung möglich (sogar notwendig) - und insofern ist unsere AkteurIn ein »AkteurIn-Subjekt« (Keller 2012: 76), dessen Agency im Kontext von Unterwerfung und Gestaltung anzusiedeln ist. Damit tragen wir dem Umstand Rechnung, dass - wie wir unten noch erläutern werden - die der pragmatistischen Theorie entlehnte kreative Handlungsfähigkeit von AkteurInnen an (diskursive) Grenzen stoßen (Nonhoff/Gronau 2012: 128). ${ }^{11}$

Die zweite Frage zielt auf unser Normverständnis. Hier ist Peter Katzensteins oben angeführte Standarddefinition von Normen als "collective expectations for the proper behavior of actors with a given identity « Katzenstein 1996: 5) für unser Unterfangen zunächst als nicht problematisch zu bezeichnen, insofern diese auf die intersubjektiv geteilte Erwartungshaltung für angemessenes Verhalten von AkteurInnen bezogen wird. Problematisch aus theoretischer und forschungspraktischer Sicht ist vielmehr die given-ness der identity. Theoretisch gesehen verengt sie den Entstehungsprozess von Identitäten, da sie als Ausfluss von Normstrukturen konzeptualisiert werden: »Thus norms either define (>constitutes) identities in the first

10 Gerade die poststrukturalistische Diskurstheorie, wie wir sie hier vertreten, ist eine der zentralen Referenzen für die Eigenmächtigkeit von AkteurIn-Subjekten innerhalb des Universums poststrukturalistischer Theorien (Keller 2012: 76). Diese Variante der Diskurstheorie firmiert unter dem Begriff Essex School of Discourse Theory; zentrale Vertreterinnen sind hier Laclau und Mouffe mit ihrem Werk Hegemony and Socialist Strategy (2001), weitere Vertreter sind Aletta Norval, David Howarth oder Jason Glynos. Mit dieser Betonung soll zugleich gesagt sein, dass die Kombination mit einer pragmatistisch inspirierten Handlungstheorie nicht so undenkbar ist, wie manch eine(r) meinen mag. Vielmehr reiht sich unser Vorschlag in die aktuell - gerade mit Blick auf die Diskurstheorie von Foucault - geführten Diskussionen ein, die »das Misstrauen gegen die Souveränität des Subjekts nicht länger als das letzte Wort in der Diskurstheorie gelten lassen wollen« (Renn 2012: 35) und in denen die Zusammenführung unter anderem in der Formel der »pragmatisierten Diskurstheorie« (Renn 2012: 35) mündet.

11 Wesentlicher Grund für ein Festhalten an dem oder der AkteurIn oder für die Reihung »AkteurIn-Subjekt « ist die Frage, ob es neben individuellen auch kollektive Subjekte geben kann; eine Problematik, die wir hier nicht diskutieren können. Vgl. dazu Epstein (2011); Howarth (2000) und den bereits genannten Beitrag von Nonhoff/Gronau (2012). 
place $[\ldots]$ or prescribe or proscribe (>regulates) behaviors for already constituted identities « (Jepperson et al. 1996: 54). So geht verloren, dass der identitätsgetriebene Rekurs auf Normen nicht unbedingt gleichbedeutend mit einem einheitlichen Verständnis dieser Normen sein muss (weswegen der Effekt von Normen eben auch nicht immer einheitlich gleich ist), obgleich Normen trotz konfligierender Interpretationen sehr wohl integrativ wirken können (wie beispielsweise Thorsten Bonacker und André Brododcz für die symbolische Integration durch Menschenrechte gezeigt haben (2001)).

Zudem wird mit der Referenz auf die given identity erneut der Blick auf Agency verstellt, denn Identifikationsprozesse entstehen aufgrund der Kontingenz diskursiver Strukturen und verlangen AkteurInnen eine beständige Entscheidung bzgl. ihrer jeweiligen Identifikation ab. Aus forschungspraktischer Sicht verleitet die given identity schließlich im Zugriff auf den Untersuchungsgegenstand die bisherige Forschung dazu, Akteursidentitäten häufig zu Konstanten zu erklären, die zu Kausal- und Subsumptionslogik passen: Weil die USA sich als zivilisierte Nation verstehen, setzen sie keine Nuklearwaffen im Krieg ein.

Historische Kontingenz und stets nur temporäre Fixierung von Identitäten und Normen stehen dem jedoch entgegen - und kritische Normenforschung sollte diesem Umstand systematisch Rechnung tragen. Wir argumentieren daher, dass Normen Teil der für AkteurInnen bandlungsleitenden Regeln sind - bis sie sich gezwungen sehen, diese zu hinterfragen. Durch Diskurse wiederum werden Normen intersubjektiv konstruiert, vorgehalten und aktualisiert - bis diese ihre Repräsentationsfunktion einbüßen. Die analytischen Konzepte Krise und Dislokation verbinden diese Momente des Umbruchs und des normativen Wandels prozessual.

\subsubsection{Dislokation}

Hat die in den IB durch poststrukturalistische Arbeiten erneuerte AkteurIn-Struktur-Debatte von Beginn auf diskurstheoretische Ansätze rekurriert, ${ }^{12}$ so haben in letzter Zeit ForscherInnen die Diskussion unter Rückgriff vor allem auf die Essex School (vgl Hansen 2006; Herschinger 2011;

12 Siehe dazu die Debatte zwischen Doty (1997; 1999; 2000) und Wight (1999; 2000). 
Renner 2013) ${ }^{13}$ entscheidend angereichert. Diese Fortführung bedeutet eine theoretische Erweiterung sowohl auf Struktur- als auch Akteursseite.

Besonders wichtig für unser Unterfangen ist zunächst die zentrale Idee der Bruchstellen im Diskurs, durch die eine Unabgeschlossenheit der Struktur entsteht. So konzeptualisieren Ernesto Laclau und Chantal Mouffe Diskurse als einen spezifischen Zusammenhang von Elementen, die mit einander in Beziehung gesetzt werden müssen, um Bedeutung zu erlangen (Laclau/Mouffe 2001: 105) - eben jene Bedeutungen, die mit in unserem Verweis auf Diskurse als »structure of meaning-in-use (Weldes/Saco 1996: 373) gemeint sind. Diese Bedeutungen kommen durch die Abgrenzung von dem zustande, was der Diskurs nicht ist: Diskurse leiden an einem unüberwindlichen Mangel, der sich aus der Notwendigkeit ihrer Abgrenzung ergibt, die nie vollständig sein kann, sondern immer wieder durch das Ausgegrenzte gestört wird. Damit "wird deutlich, warum ein Diskurs sein eigenes In-Erscheinung-treten nicht vollständig determinieren kann, sondern immer wieder durch das >Außen` gestört wird « (Keller 2012: 78).

In dieser Unabgeschlossenheit der Struktur kommt das Konzept der Dislokation zum Tragen, denn: Strukturen sind immer auch dislozierte Strukturen. Dislokation benennt das Dezentrieren einer Struktur durch soziale Prozesse. Elemente, die nicht in eine sinnhafte Beziehung gesetzt werden, also von der bestehenden Struktur nicht symbolisiert werden können, führen zu ihrer Destabilisierung. Dislokation ist andauernd, insofern als in diskursiven Strukturen, wie oben erläutert, ein unbestimmter, unsagbarer Rest verbleibt, der sich der Symbolisierung entzieht. Dislokation enthüllt die Kontingenz der Struktur: Der Moment der Dislokation ist das Trauma der Struktur, das sie unvollständig bleiben lässt, und es ist eben diese Unvollständigkeit der Struktur, die Agency ermöglicht (Laclau 1990: 41-43). Dazu unten gleich mehr.

Auch wenn die Kontingenz der Struktur im Moment der Dislokation sichtbar ist und die Unvollständigkeit der Struktur enthüllt, bedeutet Kontingenz der Struktur hier nicht, dass überhaupt keine strukturellen Festlegungen möglich sind. Eine Norm ist, wie oben angedeutet, eine solche Festlegung, d.h. die Determinierung einer kollektiven Erwartung spezifischer Praktiken und Handlungen.

Allerdings sind solche Festlegungen immer nur partielle, temporäre Fixierungen, denn "neither absolute fixity nor absolute non-fixity is possible« (Laclau/Mouffe 2001: 111). Vielmehr entstehen durch temporäre Festle-

13 Für eine erste wie umfassende Diskussion und Operationalisierung der Hegemonietheorie siehe Nonhoff (2006). 
gungen Bedeutungen und Praktiken, d.h. diskursive Strukturen. Doch bleibt dabei zu bedenken, dass jede Fixierung auch der machtgeladene Versuch ist, eine bestimmte Bedeutung, eine kollektive Erwartungshaltung als dominant zu etablieren (Laclau/Mouffe 2001: 111-112) - für AkteurInnen ist sie der Versuch, Entscheidungen, Bedeutungen, Praktiken, Handlungen in der einen und eben nicht in einer anderen Weise zu artikulieren.

Im Kontext der Normenforschung bedeutet die Dislokation, dass die bestehende Bedeutungsordnung, die structure of meaning-in-use destabilisiert wird. Geltende und gleichwohl unabgeschlossene Normstrukturen können im Moment der Dislokation nicht mehr aufrechterhalten werden, verlieren ihre Bedeutung als Fundament, als Standard für angemessenes Verhalten in bestimmten Situationen. Dislokationen dezentrieren bestehende Normstrukturen, durch sie wird Wandel dieser Normstrukturen möglich.

\subsubsection{Krise}

AkteurInnen führt eine Dislokation des Diskurses die Kontingenz und Unabgeschlossenheit diskursiver Strukturen vor Augen. Dislokation bedeutet für AkteurInnen somit Zweifel mit Blick auf ihre bisherigen Entscheidungs- und Identifikationspunkte sowie Handlungsroutinen. AkteurInnen sehen sich aufgrund dessen (aufgrund der Kontingenz und Instabilität diskursiver Strukturen) beständig dazu gezwungen, sich neu im Diskurs zu verorten, d.h. neue Identifikationspunkte zu finden. Diesem Druck zur Entscheidung, zur neuen Identifikation kann sich eine AkteurIn kaum entziehen, denn er oder sie kann sich nicht nicht entscheiden bzw. nicht nicht identifizieren (Laclau/Zac 1994). Diese Entscheidungen, Neu- und ReIdentifikationen lassen die Struktur nicht unberührt, da sie neue Strukturopportunitäten eröffnen - die erlauben, aber auch erzwingen, dass eine AkteurIn sich neu situiert, ist es doch deren Entscheidung, die den Bruch in der Struktur überwinden kann (Stäheli 2000a: 60-61).

Die dezentrierte Struktur erzwingt also die Rekonstitution, Neu- und ReIdentifikation von AkteurInnen, sprich, nach dem von uns hier vorgeschlagenen Vokabular müssen AkteurInnen handeln. Und nicht irgendwie handeln, sondern kreativ, um der Krise zu entgehen. Die pragmatistische Handlungstheorie liefert dafür den entscheidenden konzeptionellen Anknüpfungspunkt, denn sie sieht »alles menschliche Handeln $[\ldots]$ in der Spannung zwischen unreflektierten Handlungsgewohnheiten und kreativen Leistungen « (Joas 1992: 190). Was bedeutet das für das systematische Erfassen von Agency? Und warum argumentieren wir, dass gerade hier der 
Gewinn unserer Kombination von poststrukturalistischer Diskurstheorie und pragmatistisch inspirierter Agency liegt?

Agency erfolgt nach Handlungsregeln, die auf Glaubenssystemen und auf durch Erfahrung etablierte Praktiken ruhen (Hellmann 2009; 2010). ${ }^{14}$ Glaubenssysteme und Praktiken verweisen dabei wechselseitig aufeinander: »Practices could not exist if people did not have appropriate beliefs; and beliefs or meanings would not make sense in the absence of the practices to which they refer" (Bevir 2006: 284). Und Diskurs im Sinne einer structure of meaning-in-use erlaubt die Einbettung von Agency im sozialen Kontext, also über die einzelne AkteurIn hinaus. So wird sinnvolles, intersubjektiv nachvollziehbares Handeln in der sozialen Realität möglich (Hofferberth/Weber 2015: 86-88).

In Routinesituationen bedienen sich AkteurInnen bestehender, bewährter Bedeutungen und Praktiken, um sozialer Realität und den sich daraus ergebenden Handlungsoptionen Sinn zuzuschreiben und dementsprechend sinnvolle Handlungen zu vollziehen. In der Regel findet Agency in solchen Routinesituationen statt, nicht selten sogar habitualisiert. Bestehende Glaubenssysteme und Handlungsregeln wirken somit im Alltag handlungsentlastend für AkteurInnen. Folglich, weil Agency in diesem Sinne stets sozial kontextuiert und nicht selten routinisiert ist, erleben wir die soziale Realität trotz ihrer Kontingenz als - vergleichsweise - geordnet und nicht vollkommen chaotisch.

Doch Dislokation ist eben, wie oben erläutert, andauernd und wiederkehrend. Sie stellt AkteurInnen folglich vor krisenhafte Entscheidungssituation, wenn durch sie aus Akteursperspektive bestehende Routinen in besonderem Maße herausgefordert, Glaubenssysteme erschüttert, Erwartungen widerlegt und Zweifel geschürt werden. Und weil die alltäglichen Problembewältigungsmechanismen mitunter versagen, ist in der Folge kreatives, aber zugleich situationsangemessenes Handeln notwendig - also Handeln, das von Routinen abweicht, aber nichts destotrotz sinnlogisch an bestehende soziale Realität anzuknüpfen erlaubt. Agency ist nach diesem Verständnis also in einen steten Erfahrungsstrom aus Krisen und Routinen ein-

14 Wir sprechen in Bezug auf unser Konzept von Agency nur von durch den Pragmatismus inspiriert, weil wir nicht mehr als die Nutzbarmachung der für pragmatistische Handlungstheorie kennzeichnenden Krise-Routine-Dialektik beabsichtigen. Siehe für den Versuch der darüber hinaus gehenden Fruchtbarmachung des Pragmatismus für die IB im weiteren Sinne und in direkten Bezug auf Denker wie Charles Sanders Peirce und John Dewey neben den genannten Beiträgen von Hellmann auch Friedrichs (2009); Kivinen/Piiroinen (2007); Kratochwil (2007); Roos (2012); Rytövuori-Apunen (2009); Sil (2009). 
gebettet und somit immer kreatives und (sozial, diskursiv) situiertes Handeln zugleich:

»Dieses Verständnis situativen und genuin kreativen Handelns impliziert, dass es unangemessen wäre, eine Handlung als `Einzelhandlung، aus ihrem Kontext, jenem >geschlossenen oder >unterbrochenen< $\mathrm{Zu}$ sammenhang unserer Erfahrung, herauszulösen und im Sinne einer (auf diese >Einzelhandlung« reduzierten und ihr vorgelagerten) ZweckMittel-Relation zu beschreiben« (Hellmann 2010: 150).

Ist eine kreative Handlung im Zuge des Zweifels aus Akteursperspektive erfolgreich, dann werden als unwirksam und nicht zielgerichtet erfahrene Handlungsroutinen durch die problemadäquateren (im Sinne einer sinnvollen Bewältigung der Krise) ersetzt, also letztlich neue Handlungsroutinen etabliert - und der Zyklus setzt sich fort (Hellmann 2010: 150-153; Hellmann 2009: 5-12; Joas 1992: 190).

Nach dieser Krise-Routine-Dialektik kommt durch Agency also Neues verstanden nicht als wundersam-spontane Schöpfung aus dem Nichts, sondern als sozial situierte, sinnvolle, kreative Rekombination von Handlungsregeln - in die Welt. Und damit werden die notwendigen konstitutiven »Ressourcen" (Renn 2012: 39, 41) für Diskurs- und Normstrukturen bereitgestellt. Agency ist also die Entscheidung oder die Akteurshandlung, die die als krisenhaft erfahrene dislozierte Struktur zu überwinden sucht. Vor allem der Verweis darauf, eine Handlung nicht als Einzelhandlung zu isolieren, erinnert daran, dass AkteurInnen natürlich nicht nur in eine Normstruktur eingebettet sind; vielmehr sind AkteurInnen in ein Netz von Diskursen eingebunden und haben somit ein Repertoire an Strukturen zur Verfügung, aus der sie die Rekombinations-Ressourcen für die Begegnung mit der als krisenhaft erfahrenen dislozierten Struktur ziehen.

Genau darin liegt unserer Ansicht nach der Gewinn einer Kombination aus poststrukturalistischer Diskurstheorie und pragmatistisch verstandener Agency. Denn das Neue, das durch Agency in der oben erläuterten Form in die Welt kommt, ist eben nicht "auf die Wahl zwischen Möglichkeiten, die von der Struktur selbst erzeugt werden, reduziert" (Stäheli 2000b: 238). Unser Begriff von Agency geht also über die rein diskurstheoretische Argumentation hinaus, nach der die durch Dislokation eröffneten Entscheidungsmöglichkeiten »explizit als Möglichkeiten der Struktur eingeführt" (Stäheli 2000b: 238, Hervorh. dort) werden. Denn wenn nur angenommen wird, dass AkteurInnen "actualize certain structural potentialities and reject others " (Laclau 1990: 30) und dass die Entscheidung "a reaching out for the possible (Stäheli 2000b: 238) ist, dann wird das Neue auf die Hervorbringung dessen reduziert, was möglich ist - und mehr nicht. Wer, wie, 
wann, wo (sprech-)handelnd das Mögliche oder auch weniger Mögliche ergreift - also nicht irgendwie, sondern kreativ handelt -, ist damit nicht in den Blick zu bekommen. Unser Vorschlag einer pragmatistisch verstandenen Agency sorgt insofern für eine theoretische Blickerweiterung, da so im ko-konstitutiven Verhältnis von AkteurIn und Struktur auch das Neue, Unvorhergesehene, Kontestierende, »Unangemessene« (Stichwort norm entrepreneurs) erfassbar wird.

Durch die Konzeptualisierung von Strukturen als unabgeschlossene, dislozierte Strukturen und von Agency als wiederkehrender Notwendigkeit zu Entscheidungen und kreativem Handeln im Kontext der Krise-RoutineDialektik werden normativer Wandel und Normgenese nicht als zu erklärendes Sonderproblem gefasst, sondern als relationales, wiederkehrendes Moment. Durch die hier vorgeschlagene Perspektive auf Agency und Struktur werden also Mikroprozesse normativen Wandels sichtbar und so (letztendlich auch) die Etablierung neuer, intersubjektiver normativer Strukturen systematisch und in theoretisch informierter Weise rekonstruierbar. Damit ist das Stichwort für die methodologischen Implikationen dieser Perspektive gefallen.

\subsection{Zweiter Schritt: Privilegierung rekonstruktiver Forschungslogik}

Eingangs haben wir argumentiert, dass der Fokus auf die Momente der Krise und der Dislokation kritisches Potential eröffnet, da der explizite Einbezug von Agency potenziellen Widerstand gegen bestehende Normstrukturen systematisch offen zu legen erlaubt. ${ }^{15}$

Wenn wir nun plädieren, dass kritische Normenforschung stärker rekonstruktionslogisch vorgehen sollte (siehe grundlegend Herborth 2010; 2011), dann ist die Idee, dabei nicht zu konstatieren, dass die Dinge anders bätten kommen können. Das ist gleichermaßen wahr wie trivial. Der war on terror, als Beispiel, war bekanntermaßen nicht völlig alternativlos - der Widerstand von Staaten wie Deutschland oder Frankreich gegen den Irakkrieg oder das Infragestellen einer militärischen Reaktion auf die Terrorismusfrage verweisen darauf (vgl. Jackson 2005). Wenn AkteurInnen aber eben nicht als Normbefolgungsautomaten, sondern als (in Routinen wie Krisen) in einem kontingenten sozialen Bedeutungskontext genuin handelnde AkteurInnen verstanden werden (Bevir 2006: 285), dann ist der springende

15 Siehe zur Kontestation von Normen vor allem die Arbeiten von Wiener (2004; 2008). 
Punkt, dass andere Fragen an das empirische Material ermöglicht werden. Wird Agency nicht mehr als Effekt eines kausalen Wirkmechanismus verstanden, dann ist es möglich - und aus unserer Sicht sogar geboten - zu fragen, wie genau ein bestimmtes Handeln zustande kam, eben gerade weil plausiblerweise auch anders hätte gehandelt werden können. Die Idee der Rekonstruktion ist somit zu zeigen, wie genau die Dinge so zustande kamen und eben nicht anders (Herborth 2010: 273). Dies bedarf entsprechender - rekonstruktionslogisch angemessener - methodologischer und methodischer Zugriffe (siehe dazu Franke/Roos 2010).

Dies erleichtert erstens eine Forschungspraxis, die kritisch aufzeigt, wie genau AkteurInnen sich mit bestimmten Normen identifizieren und gleichermaßen von Normen vereinnahmt, verregelt werden. So kann beispielsweise die Art problematisiert werden, mit der Verfechter eines war on terrors sich in dogmatischer Weise mit einer militärischen Lösung für das $\mathrm{Si}$ cherheitsproblem Terrorismus identifizieren und diese als die scheinbar einzig mögliche Lösung für alle Probleme darstellen, die sich mit dem Terrorismus verbinden lassen, etwa der war on drugs, der war on poverty oder unlängst auch der war on migrants (vgl. für den war on terror Björnehed 2004; Jackson 2005; Noborder Network 2006). Die militärische Lösung wird zum Allheilmittel und der war on terror strukturiert unzählige andere Arten von sozialen Beziehungen und Praktiken (z.B. die zwischen privaten AkteurInnen, etwa zwischen Passagier und Fluggesellschaft), was zahlreiche Probleme aufwirft (etwa Datenschutz, Grundrechte etc.), da die militaristische Logik hier nur fremdkörperhaft funktioniert (vgl. für andere Beispiele Glynos/Howarth 2007: 197).

Zweitens kann durch den Fokus auf Dislokation und Krise die aktuelle Normenforschung zu schlechten bzw. zum Wandel von guten zu schlechten Normen befruchtet werden (vgl. Badescu/Weiss 2010; Geis et al. 2013.; Heller et al. 2012; Liese 2009; Picarelli 2009), indem sich kleinteilig erforschen lässt, wie genau AkteurInnen unter Bezug auf bestimmte Normen entscheiden und handeln (statt diesen Normen einen vorab definierten $E f-$ fekt zu unterstellen). Denn auch gute Normen ermöglichen Akteurshandeln, das problematisiert werden kann und sollte. So ließe sich mit Blick auf ein aktuelles Beispiel argumentieren, dass für Demokratien identitätsstiftende, gute Normen wie der Schutz menschlichen Lebens oder die Wahrung der Angemessenheit der Mittel beim Einsatz von militärischer Gewalt die Bedingung der Möglichkeit für aktuelle Drohnenkriegsführung bieten - eine Praxis, die paradoxerweise mit genau diesen Normen konterkarierenden Konsequenzen verbunden ist (Sauer/Schörnig 2012). 


\section{Das globale Drogenprohibitionsregime als Illustrationsbeispiel}

Wie sich unsere theoretische und forschungspraktische Alternative konkret bewähren kann, lässt sich hier am Beispiel des globalen Drogenprohibitionsregimes aus Platzgründen nur knapp illustrieren.

Im Jahr 2012 gilt: Drogen sind verboten und illegal, ihr Konsum ist ein von der gesellschaftlichen Norm abweichendes Verhalten. Die einzige strikt kontrollierte - Ausnahme: Die Pharmazie darf legal von Drogen Gebrauch machen, um Schmerz zu lindern und auch, um Abhängigen den Weg in ein drogenfreies Leben zu ermöglichen. Historisch gesehen ist dieses globale Verbot von Drogen und das damit einhergehende globale Drogenprohibitionsregime eine jüngere Entwicklung. Menschen haben über Jahrhunderte Drogen genutzt und in vielen Gesellschaften spielt(e) Drogenkonsum eine wichtige soziokulturelle Rolle. Geht man in das 19. Jahrhundert zurück, so findet man neben Limonaden und alkoholischen Getränken, angereichert mit Bestandteilen der Coca-Pflanze, Opiate als Antidepressiva, Heroin (kurioserweise) als Gegenmittel bei Morphiumsucht sowie ÄrztInnen, die das zur Verschreibung vorgesehene Morphium gerne selbst nutzen (Briesen 2005: Kap. 2).

Chemische und medizinische Innovationen machten in diesen Jahren den modernen Drogenkonsum möglich: 1805 gelang die Isolation von Morphium aus Opium, 1843 wurde die intravenöse Injektion flächendeckend durchführbar, 1855 folgte die Herstellung von Kokain aus der CocaPflanze und 1874 wurde Heroin erfunden. Während Innovationen wie Morphium, Opium und Heroin einerseits als Wundermittel gepriesen wurden, wuchs andererseits die Besorgnis vor potenziellen Schäden durch Drogen, vor allem in der britischen und der US-amerikanischen Gesellschaft (Nadelmann 1990: 504).

Konkret fühlten sich bestimmte AkteurInnen herausgefordert. Hintergrund war eine Entwicklung, die sie aus unterschiedlichen Gründen als krisenhaft empfanden: Einflussreiche MissionarInnen, die den Drogenkonsum in den zu bekehrenden Ländern und Kolonien für deren Widerstand gegen den richtigen Weg verantwortlich machten. Gesellschaftliche Gruppen wie die Anglo-Oriental Society for the Suppression of the Opium Trade von 1874 und die erstarkenden Berufsgemeinschaften der MedizinerInnen und PharmazeutInnen, die vor gesellschaftlichen Konsequenzen warnten. So entwickelte sich eine Bewegung, die das Drogen-Problem breiteren Gesellschaftsschichten vor allem durch die Kombination mit rassistischen Vorurteilen kommunizierte. Auf diese Weise wurde das Opiumrauchen chinesischer EinwanderInnen zum Symbol für die Dekadenz der MigrantInnen, zu deren Waffe für die Zerstörung der US-amerikanischen Gesellschaft. Im 
Süden der USA befürchteten Weiße zunehmend, dass der Kokainkonsum Schwarze aufsässig machen und sie ihren Platz in der sozialen Ordnung vergessen lassen würde. Von JournalistInnen, PolitikerInnen und den Strafverfolgungsbehörden aufgegriffen, wurden diese Vorurteile in gesetzliche Regelungen gegossen (vgl. Briesen 2005; Musto 1973: 6).

Zum Ende des 19. Jahrhunderts zeichneten sich diese Veränderungen des Diskurses zu Drogen deutlicher ab, getragen von gesellschaftlichen Entwicklungen, die von der bis dato bestehenden Diskursstruktur nicht symbolisiert werden konnten - war diese Struktur doch von einer liberalen, gar indifferenten Haltung gegenüber Drogen geprägt gewesen. Ansteigender Drogenkonsum, fremdenfeindliche Ängste, medizinisch-chemische Innovationen erzeugten eine Gemengelage einander widersprechender Entwicklungen, die nicht ohne weiteres in eine sinnhafte Beziehung zueinander gesetzt werden konnten und so zur Destabilisierung der alten Struktur beitrugen. Folglich entstanden die Zweifel der AkteurInnen mit Blick auf ihre bisherigen Entscheidungs- wie Identifikationspunkte und Handlungsroutinen hier im Wechselspiel mit der dislozierten Struktur.

Für unser Anliegen interessant ist an dieser Destabilisierung die sich entwickelnde Hinwendung zu einer internationalen Kontrolle und Prohibition von Drogen - etwas, das es vorher in dieser Form nicht gab. Während die geltenden Normstrukturen - kein Verbot, keine Ächtung, freier, unregulierter Umgang mit Drogen - ihre Bedeutung als Standard für angemessenes Verhalten verloren, eröffneten sich angesichts der als gesellschaftliche Krisensituation markierten Prozesse eine Kluft zu den neuen Möglichkeiten - hier vor allem ein Verbot von Drogenkonsum - für die (noch) keine Lösungsroutinen bereitstanden. Eine Kluft öffnete sich also zwischen der dislozierten Struktur und den Entscheidungen, die diesen $\mathrm{Zu}$ stand durch Agency überwinden sollten (Stäheli 2000a: 60-61). Die zunehmend auftauchenden Rufe nach einer globalen Kontrolle von Drogen können folglich in unserem Sinne als kreative Handlungen gelten, die Neues hervorbrachten. Sie waren Handeln, das von der Routine (einer Welt frei von internationalen Kontrollen für Drogen) abweicht, aber sinnlogisch zugleich an bestehende soziale Realitäten anzuknüpfen erlaubte (Drogenkonsum als gesellschaftliche Grenzen überschreitendes Regulierungsproblem - siehe Assoziation mit EinwanderInnen etc.).

Im Verlauf des 20. Jahrhunderts entwickelte sich eine immer umfassendere globale Kontrolle von Drogen, die mit der Vereinbarung der Single Convention on Narcotics Drugs 1961 unter der Ägide der Vereinten Nationen und den folgenden zwei weiteren Konventionen in ein System globaler Drogenprohibition mündete (vgl. Bruun et al. 1975; Nadelmann 1990: 
502-513). Mit der Single Convention wurde der universelle Anspruch des entstehenden Regimes hinsichtlich Produktion, Handel und Besitz deutlich. ${ }^{16}$

Mit ihr wurde die Norm - Drogen sind illegal und verboten, ihr Konsum und Handel ist deviantes Verhalten - global; und mit Folgekonventionen von 1972 und 1988 wurde sie stabilisiert. Die zügige Erweiterung des Geltungsbereichs der Prohibition (sowohl geographisch als auch bezüglich der Sachbereiche) bezeugt einerseits die beständige Festlegung, d.h. die Zementierung der Drogenprohibitionsnorm, einer kollektiven Erwartung spezifischer Praktiken und Handlungen angesichts der "source of danger to the health of the whole world « (UN 1964: 6). Diese Festlegung ist die sinnhafte Verbindung von Elementen - Drogen, Gefahr, Menschheit, Gesundheit - in eine diskursive Struktur. Andererseits ist eben diese Festlegung verstanden als die dazu führenden Entscheidungen, also Agency - als der Versuch von AkteurInnen zu verstehen, Entscheidungen und Handlungen in einer bestimmten Art und Weise und eben nicht anders zu artikulieren. Die Norm, Drogen zu verbieten, ist insofern der machtgeladene Versuch, eine bestimmte Bedeutung, eine kollektive Erwartungshaltung als dominant zu etablieren - dies auch angesichts der in den letzten beiden Jahrzehnten zunehmenden Umstrittenheit der Norm des globalen Drogenverbots mit Blick auf den war on drugs und seine fatalen Folgen. Darin liegt der grenzziehende Charakter solcher Festlegungen: Sie etablieren nicht nur die Identität der AkteurInnen, die alle inkludieren, die einem globalen Drogenverbot zustimmen, sondern kreieren dabei gleichermaßen via Exklusion die Identität derer, die gegen ein solches Verbot sind (und damit auch keine Stimme - keine Entscheidungsmöglichkeiten, keine Agency im Diskurs erlangen können) (vgl. Herschinger 2011: Kapitel 3). Damit ist gleichermaßen gesagt, dass normativer Wandel, betrachtet durch das Brennglas von Dislokationen und Krisen, nicht gleichbedeutend sein muss mit Veränderung hin zu neu im Sinne von nie dagewesen, sondern neben Widerstand auch Stabilisierungsprozesse oder die Reifizierung von Normen beinhalten kann.

Das Beispiel illustriert, wie Agency in einen steten Erfahrungsstrom von Krise und Routine eingebettet und die Rekombination von Diskursressourcen durch kreatives Handeln möglich ist. Es zeigt auch, dass eine re-

16 Die Akzeptanz des UN-Drogenregimes ist heute weltweit: 186 Staaten gehören laut United Nations Office on Drugs and Crime der 1961er Konvention, 184 Staaten der 1971er Konvention, 191 Staaten der 1988er Konvention an (Stand Januar 2021), vgl. UNODC (2021). 
konstruktive Forschungspraxis Antworten darauf bieten kann, wie die globale Drogenprohibitionsnorm so zustande kam und nicht anders.

\section{Schlussbetrachtung}

Dem Status quo der Normenforschung haben wir hier mit den beiden Konzepten Krise und Dislokation eine Alternative gegenübergestellt, um Relationalität und Ko-Konstitutivität des AkteurIn-Struktur-Verhältnisses einzufangen. Der Mehrwert dieser Alternative liegt unseres Erachtens darin, dass er mittels rekonstruktionslogischer Methoden das ko-konstitutive Wechselspiel von AkteurInnen und Struktur forschungspraktisch systematisch aufzuzeigen erlaubt, wodurch kritische Potenziale für die Normenforschung freigesetzt werden.

Uns war hier daran gelegen, ein Vokabular zu entwickeln und vorzustellen, das hilfreich, aber selbstverständlich nicht allgemeingültig anleitend, für konkrete Forschungspraxis sein kann. Insofern handelt es sich bei Krise und Dislokation also um nicht mehr - aber eben auch nicht weniger als einerseits metatheoretische Formeln für Kontingenz sowie andererseits methodologisch-methodische Heuristiken, die in konkreter, rekonstruktiver empirischer Forschung (ausführlicher als wir sie hier skizzieren konnten) sowie stets im Lichte des konkreten Untersuchungsgegenstands Empirie gesättigt mit Bedeutung gefüllt werden müssen. Was Krise und Dislokation bedeuten und wie sie das Verhältnis von Agency und Struktur hinsichtlich bestimmter Normen, ihrer Entstehung, ihrem Wandel, ihrem Verschwinden und Ersetzt-Werden artikulieren, das ist demzufolge eine Frage konkreter Forschungsarbeiten, eine Frage der Empirie.

\section{Literatur}

Adler, Emanuel 1997: Seizing the Middle Ground, in: European Journal of International Relations 3: 3, 319-363.

Badescu, Christina G./Weiss, Thomas G. 2010: Misrepresenting R2P and Advancing Norms: An Alternative Spiral?, in: International Studies Perspective 11: 4, 354-374.

Bevir, Mark 2006: How Narratives Explain, in: Yanow, Dvora/Schwartz-Shea, Peregrine (Hrsg.): Interpretation and Method, Armonk, NY, 281-290.

Björnehed, Emma 2004: Narco-Terrorism: The Merger of the War on Drugs and the War on Terror, in: Global Crime 6: 3-4, 305-324. 
Bonacker, Thorsten/Brodocz, Andre 2001: Im Namen der Menschenrechte. Zur symbolischen Integration der internationalen Gemeinschaft durch Normen, in: Zeitschrift für Internationale Beziehungen 8: 2, 179-208.

Briesen, Detlef 2005: Drogenkonsum und Drogenpolitik in Deutschland und den USA. Ein historischer Vergleich, Frankfurt a. M..

Bruun, Ketill/Lynn, Pan/Rexed, Ingmar 1975: The Gentlemen's Club: International Control of Drugs and Alcohol, Chicago, IL.

Bucher, Bernd 2014: Acting abstractions: Metaphors, narrative structures, and the eclipse of agency, in: European Journal of International Relations 20: 3, 742-765.

Chayes, Abram/Chayes, Antonia Handler 1994: Regime Architecture: Elements and Principles, in: Nolan, Janne E. (Hrsg.): Gobal Engagement. Cooperation and Security in the 21st Century, Washington, DC, 65-131.

Davis, James W. 2005: Terms of Inquiry, Baltimore, MD.

Deitelhoff, Nicole 2009: The Discursive Process of Legalization, in: International Organization 63: 1, 33-65.

Doty, Roxanne Lynn 1997: Aporia: A Critical Exploration of the Agent-Structure Problematique in International Relations Theory, in: European Journal of International Relations 3: 3, 365-392.

Doty, Roxanne Lynn 1999: A Reply to Colin Wight, in: European Journal of International Relations 5: 387-390.

Doty, Roxanne Lynn 2000: Desire All the Way Down, in: Review of International Studies 26: 1, 137-139.

Epstein, Charlotte 2011: Who Speaks? Discourse, the Subject and the Study of Identity in International Politics, in: European Journal of International Relations 17: 2, 327-350.

Finnemore, Martha/Sikkink, Kathryn 1998: International Norm Dynamics and Political Change, in: International Organization 52: 4, 887-918.

Franke, Ulrich/Roos, Ulrich 2010: Rekonstruktionslogische Forschungsansätze, in: Masala, Carlo/Sauer, Frank/Wilhelm, Andreas (Hrsg.): Handbuch der Internationalen Politik, Wiesbaden, 285-303.

Friedrichs, Jörg 2009: From Positivist Pretense to Pragmatic Practice, in: International Studies Review 11: 3, 645-648.

Friedrichs, Jörg/Kratochwil, Friedrich 2009: On Acting and Knowing, in: International Organization 63: 04, 701-731.

Geis, Anna/Müller, Harald/Schörnig, Niklas (Hrsg.) 2013: The Janus Face of Liberal Democracies: Militant „Forces for Good“, Cambridge.

Glynos, Jason/Howarth, David 2007: Logics of Critical Explanation in Social and Political Theory, London.

Hansen, Lene 2006: Security as Practice, London.

Heller, Regina/Kabl, Martin/Pisoiu, Daniela 2012: The 'Dark' Side of Normative Argumentation, in: Global Constitutionalism 1: 2, 278-312.

Hellmann, Gunther 2009: Beliefs as Rules for Action, in: International Studies Review 11: 3, 638-641. 
Hellmann, Gunter 2010: Pragmatismus, in: Masala, Carlo/Sauer, Frank/Wilhelm, Andreas (Hrsg.): Handbuch der Internationalen Politik, Wiesbaden, 148-181.

Herborth, Benjamin 2004: Die via media als konstitutionstheoretische Einbahnstraße, in: Zeitschrift für Internationale Beziehungen 11: 1, 61-87.

Herborth, Benjamin 2010: Rekonstruktive Forschungslogik, in: Masala, Carlo/Sauer, Frank/Wilhelm, Andreas (Hrsg.): Handbuch der Internationalen Politik, Wiesbaden, 265-284.

Herborth, Benjamin 2011: Methodenstreit - Methodenzwang - Methodenfetisch, in: Zeitschrift für Internationale Beziehungen 18: 2, 137-151.

Herschinger, Eva 2011: Constructing Global Enemies, Abingdon.

Hofferberth, Matthias/Weber, Christian 2015: Lost in Translation. A Critique of Constructivist Norm Research, in: Journal of International Relations and Development 18: 1, 75-103.

Howarth, David 2000: Discourse, Buckingham.

Hurrell, Andrew 2002: Norms and Ethics in International Relations, in: Carlsnaes, Walter/Risse, Thomas/Simmons, Beth A. (Hrsg.): Handbook of International Relations, London, 137-154.

Jackson, Richard 2005: Writing the War on Terrorism, Manchester.

Jepperson, Ronald L./Wendt, Alexander/Katzenstein, Peter J. 1996: Norms, Identity and Culture in National Security, in: Katzenstein, Peter J. (Hrsg.): The Culture of National Security, New York, NY, 33-75.

Joas, Hans 1992: Die Kreativität des Handelns, Frankfurt a.M..

Kärger, Caroline/Kursawe, Janet/Lambach, Daniel 2017: Von Agenten, Akteuren und Strukturen in den Internationalen Beziehungen, in: Zeitschrift für Internationale Beziehungen 24: 2, 91-120.

Katzenstein, Peter J. 1996: The Culture of National Security, New York, NY.

Keller, Reiner 2012: Der menschliche Faktor, in: Keller, Reiner/Schneider, Werner/ Viehöver, Willy (Hrsg.): Diskurs - Macht - Subjekt, Wiesbaden, 69-107.

Kier, Elizabeth 1997: Imagining War, Princeton, NJ.

King, Gary/Keohane, Robert O./Verba, Sidney 1994: Designing Social Inquiry, Princeton, NJ.

Kivinen, Osmo/Piiroinen, Tero 2007: Sociologizing Metaphysics and Mind, in: Human Studies 30: 2, 97-114.

Klotz, Audie 1995: Norms in International Relations, Ithaca, NY.

Kratochwil, Friedrich 2007: Of False Promises and Good Bets, in: Journal of International Relations and Development 10: 1-15.

Kratochwil, Friedrich/Ruggie, John Gerard 1986: International Organization: A State of the Art on an Art of the State, in: International Organization 40: 4, 753-775.

Kurki, Milja 2006: Causes of a Divided Discipline, in: Review of International Studies 32: 02, 189-216.

Laclau, Ernesto 1990: New Reflections on the Revolution of Our Time, in: Laclau, Ernesto (Hrsg.): New Reflections on the Revolution of Our Time, London, 3-87. 
Laclau, Ernesto/Mouffe, Chantal 2001: Hegemony and Socialist Strategy, London.

Laclau, Ernesto/Zac, Lilian 1994: Minding the Gap, in: Laclau, Ernesto (Hrsg.): The Making of Political Identities, London, 11-39.

Legro, Jeffrey W. 1997: Which Norms Matter?, in: International Organization 51: 1, 31-63.

Liese, Andrea 2009: Exceptional Necessity, in: Journal of International Law and International Relations 5: 1, 17-47.

March, James G./Olsen, Johan P. 1998: The Institutional Dynamics of International Political Orders, in: International Organization 52: 4, 943-969.

McKeown, Ryder 2009: Norm Regress, in: International Relations 23: 5, 5-25.

Musto, David 1973: The American Disease, New Haven, CT.

Nadelmann, Ethan A. 1990: Global Prohibition Regimes, in: International Organization 44: 4, 479-526.

Noborder Network 2006: Crossing Borders, in: Transnational Newsletter 1 [http:// www.noborder.org/crossing_borders_; 27.10.2020].

Nonhoff, Martin 2006: Politischer Diskurs und Hegemonie, Bielefeld.

Nonhoff, Martin/Gronau, Jennifer 2012: Die Freiheit des Subjekts im Diskurs, in: Keller, Reiner/Schneider, Werner/Viehöver, Willy (Hrsg.): Diskurs - Macht Subjekt, Wiesbaden, 109-130.

Panke, Diana/Petersohn, Ulrich 2012: Why International Norms Disappear Sometimes, in: European Journal of International Relations 18: 4, 719-742.

Picarelli, John T. 2009: Enabling Norms and Human Trafficking, in: Friman, Richard H. (Hrsg.): Crime and the Global Political Economy, Boulder, CO., 85101.

Renn, Joachim 2012: Nicht Herr im eigenen Hause und doch nicht eines anderen Knecht, in: Keller, Reiner/Schneider, Werner/Viehöver, Willy (Hrsg.): Diskurs Macht - Subjekt, Wiesbaden, 35-51.

Renner, Judith 2013: Discourse, Normative Change and the Quest for Reconciliation in Global Politics, Manchester.

Risse, Thomas/Ropp, Steven C./Sikkink, Kathryn (Hrsg.) 1999: The Power of Human Rights, Cambridge.

Roos, Ulrich 2012: Deutsche Außenpolitik nach der Vereinigung, in: Zeitschrift für Internationale Beziehungen 19: 2, 7-40.

Rosert, Elvira/Schirmbeck, Sonja 2007: Zur Erosion internationaler Normen, in: Zeitschrift für Internationale Beziehungen 14: 2, 253-287.

Rytövuori-Apunen, Helena 2009: Abstractive Observation as the Key to the "Primacy of Practice", in: International Studies Review 11: 3, 641-645.

Sauer, Frank/Schörnig, Niklas 2012: Killer Drones, in: Security Dialogue 43: 4, 363-380.

Sending, Ole 2002: Constitution, Choice and Change, in: European Journal of International Relations 8: 4, 443-470.

Shannon, Vaughn P. 2005: Wendt's Violation of the Constructivist Project, in: European Journal of International Relations 11: 4, 581-587. 
Sil, Rudra 2009: Simplifying Pragmatism, in: International Studies Review 11: 3, 648-652.

Stäheli, Urs 2000a: Poststrukturalistische Soziologien, Bielefeld.

Stäheli, Urs 2000b: Sinnzusammenbrüche, Weilerswist.

Tannenwald, Nina 1999: The Nuclear Taboo, in: International Organization 53: 3, 433-468.

Tannenwald, Nina 2005a: Ideas and Explanation, in: Journal of Cold War Studies 7: 2, 13-42.

Tannenwald, Nina 2005b: Stigmatizing the Bomb, in: International Security 29: 4, 5-49.

Tannenwald, Nina 2007: The Nuclear Taboo, Cambridge.

UNODC 2021: Drug-Related Treaties, in: https:/www.unodc.org/unodc/en/treaties/ index.html; 25.01.2021.

Weldes, Jutta/Saco, Diana 1996: Making State Action Possible, in: Millennium: Journal of International Studies 25: 2, 361-395.

Wendt, Alexander 1999: Social Theory of International Politics, Cambridge.

Wiener, Antje 2004: Contested Compliance, in: European Journal of International Relations 10: 2, 189-234.

Wiener, Antje 2008: The Invisible Constitution of Politics, Cambridge.

Wight, Colin 1999: They Shoot Dead Horses Don't They?, in: European Journal of International Relations 5: 1, 109-142.

Wight, Colin 2000: Interpretation All the Way Down?, in: European Journal of International Relations 6: 423-430.

Yee, Albert S. 1996: The Causal Effect of Ideas on Policies, in: International Organization 50: 1, 69-108. 
